\title{
EL PROCESO CIVIL COMO JUEGO NO REPETITIVO Y COMO VÍA PARA INTERIORIZAR CARGAS INFORMATIVAS: UNA MIRADA DESDE EL ANÁLISIS ECONÓMICO DEL DERECHO
}

\author{
CIVIL PROCEDURE AS A NON-REPETITIVE MOVE AND AS A \\ MEANS OF INTERNALISING BRIEFING LOADS: A VIEW FROM THE \\ ECONOMIC ANALYSIS OF LAW
}

\section{Nicolás Carrasco Delgado*}

\begin{abstract}
RESUMEN: En este trabajo nos enfocaremos en el examen del proceso civil como contexto decisional donde es posible aplicar el análisis económico del derecho. Al respecto, no pretendemos agotar la discusión acerca de si el análisis económico del derecho y los criterios de eficiencia (por los que adquiere contenido) poseen reconocimiento en el sistema de derecho continental, o bien, si son admisibles como criterios de decisión. El propósito de este artículo es más sencillo. Buscamos señalar las razones por las que en un proceso civil, se generan los incentivos apropiados para interiorizar el costo de obtener un pronunciamiento favorable, produciéndose la información suficiente para resolver asuntos donde la eficiencia sea reconocida como factor de decisión. Esto conduce a romper la creencia habitual de que la eficiencia exige que el proceso genere tal cúmulo de información que se hace imposible su reconocimiento como factor de decisión.
\end{abstract}

Palabras clave: Análisis Económico del Derecho - Derecho Procesal - Carga de la Prueba.

\begin{abstract}
During this paper, we will focus on the civil procedure exam as a decisional context in which it is possible to apply academic analysis of law. In that regard, we do not seek to exhaust the debate as to whether economic analysis of law and efficiency criteria (for which content is acquired) enjoy recognition in the system of Civil Law or are admissible as decision criteria. The purpose of this article is more straightforward. We seek to indicate the reasons why in those civil proceedings in which efficiency is admissible as a decisional factor, the appropriate incentives are generated to internalize the cost of obtaining a favourable pronouncement, producing sufficient information to resolve the issues in which efficiency is recognised as a decisional factor. This leads to the discrediting of the habitual belief that efficiency demands that the proceedings generate enough information to make its recognition as a decisional factor impossible.
\end{abstract}

Key words: Economic Analysis of Law - Procedural Law - Burden of proof.

\footnotetext{
* Abogado. Licenciado en Ciencias Jurídicas de la Universidad de Chile. Magíster con mención en Derecho Económico de la Universidad de Chile. Doctorando en Derecho Universidad Autónoma de Madrid. Profesor de Derecho Procesal Universidad de Chile. Becario de Becas Chile para estudios en el Extranjero. Dirección Postal: Pío Nono 1, Providencia. Dirección electrónica: ncarrascod@gmail.com.
} 


\section{A MODO DE INTRODUCCIÓN: LA EFICIENCIA COMO FACTOR DE DECISIÓN JUDICIAL EN CIERTOS SUBCONJUNTOS NORMATIVOS.}

He sostenido ${ }^{1}$ que la eficiencia es un factor de decisión judicial en subconjuntos normativos determinados (por ejemplo, derecho procesal concursal ${ }^{2}$, libre competencia ${ }^{3}$, ciertas regulaciones procesales ${ }^{4}$ y sectores económicos regulados 5 ). También sostengo que en tales subconjuntos normativos, a falta de una especificación legal de la eficiencia, esta adquiere contenido conceptual por medio del criterio Kaldor-Hicks ${ }^{6}$. Pues bien, considerando tales premisas es que podemos señalar que la eficiencia-conceptualizada por medio del criterio Kaldor-Hicks-, constituye un instrumento apropiado para alcanzar objetivos de mayor bienestar social, en los subconjuntos normativos en que ese objetivo se persigue.

Como sabemos Nicholas Kaldor formuló una regla de decisión de elección social que pretendía elegir entre dos situaciones en base a un criterio que significara una mejoría a Pareto. En este sentido, propuso lo siguiente: Un estado de cosas, X, es eficiente en términos de Kaldor, a otro estado de cosas, Z, si después de moverse de Z a X, los ganadores pueden compensar a los perdedores ${ }^{7}$. Se ha complementado el criterio de Nicholas Kaldor, con lo expuesto por John Hicks cuya propuesta está sostenida en términos de la cuestión de si los perdedores podrían sobornar a los ganadores para no permitir un movimiento hacia el nuevo estado social. O sea, el perdedor pudiera estar dispuesto a pagar al ganador, una cantidad de dinero determinada para impedir el paso al nuevo estado social X, en la medida que dicha suma de dinero sea menor que la pérdida que sufre en el paso del estado social $\mathrm{Z}$ a $\mathrm{X}^{8}$.

La principal crítica a tal formulación la constituye la denominada paradoja de Scitovsky9. La misma supone una inconsistencia lógica del mencionado criterio, toda vez que, dos situaciones sociales (X, Y) pueden paralelamente cumplir con una eficiencia según Kaldor-Hicks. De esta forma, tal criterio carecería de transitividad. En efecto, puede ser que tanto $\mathrm{X}$ sea más eficiente que $\mathrm{Y}$, y asimismo, que $\mathrm{Y}$ sea más eficiente que $\mathrm{X}$, en ambos casos según Kaldor Hicks.

\footnotetext{
1 Confrontar con Carrasco y NúNez (2015) pp. 595-613; Carrasco y NúŃEz (2014) pp.30-53.

Véase, Cornelli y Felli (1997) pp. 476-477; Hart (2000) p. 18; Aghion et al. (1992) pp. 523546; Peinado (2006) p. 239; Cabrillo (1988) p.45.

3 Véase, Tapia y Cordero (2015) p. 53; Montt (2010) p. 1; Agüero et al. (2013) pp. 3-6.

Carrasco (2010) pp. 171-185.

5 Por ejemplo, en la normativa sobre fijación tarifaria de servicios sanitarios, contenida en DFL $\mathrm{N}^{\circ} 70$ de 1988, su Título I señala que se encontrarán "sujetos a fijación de tarifas los servicios de agua potable y de alcantarillado de aguas servidas, prestados por servicios públicos y empresas de servicio público, en adelante, prestadores tanto a usuarios finales, como a otros que actúen como intermediarios respecto de aquellos". Tal normativa establece de manera explícita que la finalidad del proceso de determinación de valores por la prestación de servicios sanitarios es conducir al establecimiento de una tarifa "eficiente". En este sentido, los artículos 4 y 8 del DFL N o 70 de 1988.

6 Carrasco (2015) pp. 1-15.

KALDOR (1939) pp. 549-552.

8 Confrontar con Hicks (1939) pp. 696-712; HicKs (1941) p. 108

9 Scitovisky (1941) pp. 77-88.
} 
En este sentido, planteada la crítica al criterio Kaldor-Hicks en razón de la paradoja de Scitovsky, es que muchos autores han entendido que tal criterio de eficiencia pierde toda validez como parámetro que pueda autorizar o fundar la aplicación del análisis económico del derecho normativo ${ }^{10}$. En trabajos previos he desarrollado las razones por las cuales no procede la aplicación de esta paradoja en el proceso civil ${ }^{11}$.

Con estas breves notas preliminares profundizaremos en un aspecto de la crítica al análisis económico del derecho en el trabajo de Korhnauser. La misma considera la incapacidad del proceso judicial para resolver en base a criterios de eficiencia. Daremos por establecido, en base a lo señalado, que los criterios de eficiencia son pautas de decisión que pueda usar un juez para resolver conflictos civiles en los subconjuntos normativos a los que hicimos referencia.

\section{LA CRÍTICA DE KORNHAUSER AL ANÁLISIS ECONÓMICO DEL DERECHO POSITIVO Y LA SUPUESTA IMPOSIBILIDAD DE LOS JUECES PARA FALLAR EN BASE A CONSIDERACIONES DE EFICIENCIA}

Kornhauser basa parte de su crítica al análisis económico del derecho positivo, en que el procedimiento judicial está pobremente estructurado para promover el objetivo de la maximización del bienestar ${ }^{12}$.

Cuestiona la habilidad de los jueces para resolver utilizando criterios de eficiencia, señalando que los tribunales poseen escasas competencias para hacer juicios económicos respecto de las pruebas presentadas. Los jueces no poseerían preparación para un razonamiento económico adecuado o para la manipulación de datos estadísticos, a lo que se debe sumar que generalmente tienen poco o ningún acceso a las personas que sí son entrenadas en esas materias. Agrega que en las decisiones judiciales raramente se valora el beneficio marginal de una variedad de reglas en relación a su respetivo costo marginal. Por tanto, un sistema judicial diseñado para maximizar el bienestar debería traducirse en un sistema significativamente diferente del actual ${ }^{13}$.

Tal crítica se estructura como un cuestionamiento a la perspectiva positiva del análisis económico del derecho. Urge responder, entonces ¿qué entiende Kornhauser por reclamo positivo?, al respecto indica que corresponde a aquella posición que valora que la ley es eficiente en su aplicación práctica. Al respecto, Kornhauser nos indica que para admitir tal posición, el análisis económico del derecho positivo debe hacerse cargo de tres problemas. Primeramente debe especificar el criterio de eficiencia que la ley dice adherir. Seguidamente, debe indagar si la teoría económica busca predecir aquello que la ley dice que es eficiente. Finalmente, debe examinar el mundo real para determinar si la regla legal o judicial logra en los hechos satisfacer los elementos de algún criterio de eficiencia ${ }^{14}$.

10 Véase, Coleman (2004) p. 23; Coleman (1979-1980) pp. 525-526.

11 Carrasco (2016) p. 24; Carrasco (2015) pp. 1-15.

12 Kornhauser (1980) p. 606.

13 Kornhauser (1980) pp. 606-607.

14 Kornhauser (1980) p. 610. 
En lo que sigue en este artículo, nos concentraremos en la tercera cuestión expuesta por Kornhauser. Específicamente trataremos de describir a qué se refiere tal exigencia y si es efectivo que el análisis económico del derecho no puede cumplir con la carga de que la eficiencia sea reconocida como criterio para resolver conflictos civiles.

En cuanto a la descripción de la tercera cuestión señalada por el autor, este indica que es posible dividir en dos las posiciones positivas del análisis económico del derecho: la posición evolucionaria y la posición no evolucionaria. La primera sostendría la existencia de una tendencia del sistema legal hacia la eficiencia. A pesar que algunas veces los tribunales aplican normas ineficientes, el sistema se dirige a que las reglas eficientes persistan en el tiempo en una proporción mayor que las reglas ineficientes, de forma que las primeras terminan reemplazando sucesivamente a las segundas. Por su parte, la perspectiva descriptiva no evolucionaria, simplemente considera que las reglas existentes son más eficientes que algunas otras clases de reglas.

Pues bien, dentro del análisis de las tesis evolucionarias del análisis económico del derecho positivo, Kornhauser se concentra en el proceso de toma de decisión del adjudicador ${ }^{15}$. Indaga en las razones que sugieren que ese proceso no estaría justificado por la tesis evolucionaria del análisis económico del derecho positivo. Al respecto, constata que el lenguaje de los jueces es excepcionalmente económico. Además, el proceso de adjudicación no entregaría la suficiente información a los jueces para realizar una ponderación de naturaleza económica ${ }^{16}$.

Con todo, Kornhauser no desestima que el análisis económico del derecho positivo pueda tener alguna utilidad. En este sentido, indica que la descripción que realiza el análisis económico del derecho sirve para los efectos de predecir el impacto de las reglas, toda vez que, subyace en ese análisis una relación de lógica interna entre la ley y el comportamiento de las personas en el mundo real. Dado que las reacciones de los individuos dependen de una serie de motivos y de sus preferencias, el pronóstico puede ser difícil, frente a lo cual el análisis económico permite elaborar pautas predictivas. Ello puede ocurrir en el derecho económico pero probablemente no en el derecho de familia ${ }^{17}$.

Sin entrar en el detalle de las críticas de Kornhauser debo señalar dos comentarios que vinculan tales críticas con lo tratado en este artículo: (i) En primer lugar, Kornhauser al referir a los usos descriptivos del análisis económico del derecho implícitamente sostiene que tal herramienta es relevante en ciertas áreas jurídicas y en otras no. Esa idea es coincidente con la aplicación del análisis económico del derecho que se ha planteado en la introducción, en cuanto que tal disciplina aplica en ciertos subconjuntos normativos; (iii) En segundo lugar, una de las cuestiones transversales en la crítica de Kornhauser es que existen problemas de información para que los jueces puedan hacer aplicación del análisis económico del derecho normativo y positivo. Tal objeción será tratada en lo sucesivo.

Para ello trataremos de desestimar la posición de Kornhauser. Se indicará, por tanto, que los criterios de eficiencia pueden ser aplicados por los jueces al momento de decidir en

\footnotetext{
15 Kornhauser (1980) pp. 628-629.

16 Kornhauser (1980) p. 629.

17 Kornhauser (1980) pp. 635-639.
} 
ciertos subconjuntos normativos, y que el cúmulo de información requerido para poder decidir no alcanza un nivel cuantitativa ni cualitativamente exigente como el señalado por tal autor. Para desarrollar tales ideas entenderemos el proceso judicial civil como un juego no repetitivo. A esto último nos enfocaremos en las dos secciones siguientes.

\section{EL PROCESO CIVIL COMO UN JUEGO}

El derecho procesal civil se preocupa, entre otras cosas, del procedimiento que es necesario para el conocimiento y resolución de ciertos conflictos de relevancia jurídica. Tales procedimientos corresponden a contextos de decisión social. De acuerdo a lo que hemos asumido en este artículo, en tales contextos, es posible que el juez aplique consideraciones de análisis económico del derecho. Ahora bien, cuando nos referimos a contextos de decisión social estamos pensando en el proceso como una secuencia de actos que tienden a una decisión razonada y legitimada en el respeto de ciertas reglas adjetivas ${ }^{18}$.

Es en el proceso donde el juez adopta su decisión. Son las reglas de procedimiento las que determinan las posibilidad de información y conocimiento que posee el juez respecto de los hechos; son esas mismas reglas, las que establecen restricciones al juez para ejercer sus atribuciones de la manera más óptima posible ${ }^{19}$, esto es, resolver los asuntos con la mayor celeridad y con la mayor precisión posible.

En el desarrollo de este artículo nos referiremos reiteradamente a la idea del proceso como un juego. En esa lógica, el proceso corresponde a una actividad encaminada a un fin, en el que intervienen distintos sujetos, en el marco de una relación procesal ${ }^{20}$, donde cada uno de ellos tienen un rol y busca alcanzar una decisión, ya sea, porque es su función (juez), o bien, porque afecta sus intereses (partes).

Tal idea ha sido cuestionada por algunos autores de Derecho Procesal, quienes han negado que se pueda hablar de juego procesal o de alguna metáfora similar, señalando que: "El proceso judicial, de hecho, no es asimilable a un juego. Entre las muchas razones por las que un proceso no puede considerarse un juego, la más importante de ellas es que los procesos no terminan como los juegos, las apuestas o las competiciones, ni siquiera como terminan los procesos electorales u otros tipos de pure procedural justice. Un juego contiene en sí mismo (es decir: en sus reglas de procedimiento), el mecanismo que predetermina su resultado (...) En todos estos casos no se necesita de nada más que determina de quien es la victoria o la derrota (...) El proceso en cambio, no tiene en sí mismo -es decir, en las reglas de procedimiento- mecanismo alguno que predetermine su resultado aunque, obviamente,

\footnotetext{
18 TARuffo (2002) pp. 425.

19 "Las reglas procesales imponen así ciertas restricciones a la toma de decisiones por parte del tribunal que no serían respetadas por los apostadores profesionales. Las restricciones son casi siempre más estrictas en los países del derecho común cuando los juzgados conocen de casos, que en los países del derecho civil no tienen jurados" "Las reglas del procedimiento a veces contradicen a las reglas económicas de la toma de decisiones en incertidumbre, pero existen amplias áreas de acuerdo (...) Las reglas procesales prescriben un marco cuya justificación no es necesariamente económica, pero dentro de ese marco puede operar la lógica económica” confrontar CooTER Y ULEN (2002) p. 483.

20 Véase, MARinoni et al. (2010) pp. 359-364; Von BÜlow (2008) pp. 9-16.
} 
el desarrollo concreto y específico de todo proceso condiciona de varias maneras el contenido de la decisión final”21.

Sin embargo, tal conceptualización del juego procesal es equivocada. Nadie seriamente ha comparado el juego procesal con el ajedrez, donde, por ejemplo, dadas ciertas situaciones y movimientos, se produce algo denominado "jaque mate". Por el contrario, cuando se dice que el proceso es un juego se pretende resaltar exclusivamente el carácter dirimente de las decisiones jurisdiccionales, en contraposición a las decisiones inmanentes. La cita mencionada, al criticar la idea del proceso como un juego, entiende erradamente la conceptualización de tal juego como uno de carácter inmanente.

Para efectos de clarificar ciertos conceptos, entenderemos por decisiones inmanentes aquellas que no alteran la estructura y las reglas del juego, porque simplemente las aplican (por ejemplo, las reglas que determinan el efecto de "jaque mate", cuando se producen determinados movimientos en el ajedrez). En cambio, las decisiones dirimentes son aquellas que reflejan la idea del Derecho como una actividad dinámica que se encuentra en creación constante, una de cuyas fuentes es precisamente el ejercicio jurisdiccional donde se produce la creación de normas jurídicas particulares ${ }^{22}$.

Examinaremos a continuación la idea del proceso civil como un juego dirimente no repetitivo. Ello sentará las bases para establecer un modelo, que se desarrollará en la sección 5 de este artículo, donde se explicaran las razones por las que el proceso civil como juego dirimente no repetitivo establece incentivos para el traslado al proceso de información suficiente para resolver conflictos jurídicos en ciertos subconjuntos normativos donde opera la eficiencia.

\section{EL PROCESO CIVIL COMO JUEGO NO REPETITIVO}

A continuación estudiaremos la característica del litigio como juego generalmente no repetitivo, esto es, como juego único. Su objetivo es dar cuenta del hecho que el juez al momento de adoptar una decisión judicial posee una única oportunidad para poder resolver. Esa única oportunidad de decisión es una restricción que impone el proceso al nivel de conocimiento que el juez tendrá de los hechos relevantes del asunto.

Tal factor de realidad influye en la aceptación de la eficiencia como factor de decisión en el proceso civil. En efecto, la caracterización del proceso como un juego en general no repetitivo se agrega a las reglas de convicción del proceso civil y a las normas de estándar de prueba, a fin de desestimar la procedencia de la principal crítica planteada al criterio Kaldor-Hicks como factor de decisión judicial (paradoja de Scitovsky). Lo anterior no es baladí, toda vez que, tal criterio es el que da contenido a la eficiencia en los subconjuntos normativos en donde ella es aceptada como pauta de decisión, ya sea en calidad de norma o de principio $^{23}$.

\footnotetext{
21 TARUfFo (2003) pp. 27-28.

22 Sierra Alta-Ríos (2010) pp. 379-380.

23 Se estima que los jueces en el derecho continental pueden resolver los casos que conocen aplicando consideraciones de eficiencia: (i) En aquellos supuestos en donde la normativa de manera explícita establezca la procedencia de criterios de eficiencia, y (ii) En aquellos supuestos donde la eficiencia aun cuando no es reconocida explícitamente en un texto legal, aplica como principio. Puede verse: CarrasCo y Núñez (2015) pp. 605-606.
} 
$\mathrm{Al}$ respecto, hemos sostenido que sobre la base de considerar la información imperfecta que dispone el juez para resolver un asunto -motivado por la imposibilidad de alcanzar una verdad material ${ }^{24}$-, es que el juez nunca podrá valorar los extremos de intransitividad y la falta de cardinalidad que impone la paradoja de Scitovsky como crítica de validez al criterio Kaldor-Hick ${ }^{25}$.

Sobre tales asunciones, desarrollaremos razones adicionales por las cuales tal paradoja no es procedente. Lo anterior, enmarcado en las restricciones institucionales que impone el proceso, pero no pensando en la información imperfecta de que dispone el juez para fallar, sino que, en la oportunidad única que tiene el juez para poder decidir.

Pues bien, quienquiera que pretenda seguir sosteniendo la invalidez del criterio Kaldor-Hicks en razón de la paradoja de Scitovsky en el marco específico del derecho procesal, tendrá que reconocer que sus esfuerzos se desvanecen cuando constata que Scitovsky planteó su crítica pensando en un contexto de decisión social que tuviera juego repetitivos. Dado que el proceso no corresponde a uno de aquellos contextos de decisión social, ergo, no puede admitirse en su interior una paradoja que pretende invalidar la aplicabilidad del criterio Kaldor-Hickes.

A Scitovsky le preocupaba la manera de evitar que la paradoja se produjera. Por lo mismo, formuló dos principios por medio de los cuales buscaba evitar su existencia. Tales principios, con todo, debían regir en juegos repetitivos, esto es, juegos sucesivos en donde fueran aplicados.

Por medio de esos juegos repetitivos se buscaba impedir la formulación de proposiciones de bienestar social que no cumplieran los dos principios que tal autor exigía al efecto. El primer principio, supone que la nueva decisión social genere, a lo menos, el mismo estado social inicial. Por lo tanto, no habrá oposición a que tal nuevo estado social se produzca (similar al criterio de Kaldor, pero con compensación real). El segundo principio, supone que de no ser posible lo anterior, entonces, se deberá buscar la posición superior en el nuevo estado social ${ }^{26}$ (similar al criterio de Hicks, en cuanto dicha posición superior correspondería al estado social existente luego del pago de los perdedores a los ganadores para evitar el tránsito al nuevo estado social).

Podemos explicar la operatividad de los mencionados principios de la siguiente forma:

a. Primer Principio: Concurren 2 estados sociales (X, Y), en términos tales que el estado social X es anterior al estado social Y. Ambos estados sociales son distintos ${ }^{27}$. Pero son valorados, igualmente por los sujetos implicados, por tanto, ambos son igualmente preferidos por ambos sujetos:

b. Segundo Principio: De no lograrse que el nuevo estado social Y, sea valorado al menos igualmente por los sujetos implicados, se debe buscar la subordenación social $\mathrm{Y}$, que se acerque de mayor forma al estado social X. En términos tales que si las subordenaciones sociales $\mathrm{Y}$, son $\left(\mathrm{Y}_{1}, \mathrm{Y}_{2}, \mathrm{Y}_{3} \ldots \mathrm{Y}_{\mathrm{n}}\right)$, y se da el caso que $\left(\mathrm{Y}_{1}>\mathrm{Y}_{2} ; \mathrm{Y}_{3}>\right.$

\footnotetext{
24 En este sentido, ver las razones acerca de la inexistencia de la verdad material y la imposibilidad del acceso a la misma en: TARUFFO (2002) pp. 27-57.

25 Jhunjhunwala (1974) pp. 495-496.

26 Scitovisky (1941) pp. 86-87.

27 Ello significaría que tanto X como Y se ubican en distintos puntos de una misma curva de indiferencia, según lo cual, todas las combinaciones contenidas en la curva son igualmente valorados por el sujeto en cuestión.
} 
$\left.\mathrm{Y}_{4}(\ldots) \mathrm{Y}_{\mathrm{n}-1}>\mathrm{Y}_{\mathrm{n}}\right)$, por transitividad $\left(\mathrm{Y}_{1}>\mathrm{Y}_{\mathrm{n}}\right)$, por tanto: $\mathrm{Y}_{1}$, es la subordenación social $Y$ que más se acerca al estado social $X$. De esta forma, en aplicación del segundo principio de Scitovsky, debería escogerse: $\mathrm{Y}_{1}$.

Tales juegos repetitivos surgen cuando Scitovsky exige que una decisión social deba ser adoptada analizando siempre y en todos los casos, los dos principios anteriores ${ }^{28}$. Por el contrario, para Kaldor, bastaría examinar simplemente el primero de esos principios, ya que de cumplirse, la distribución que iguala el estado social inicial constituiría un símil de la compensación real de los ganadores a los perdedores, aun cuando dicha compensación no fuera exigible.

Pues bien, para Scitovsky si el primer principio es posible, y no el segundo, entonces, la nueva situación social será mejor; si el primer principio es imposible, y el segundo principio es posible, entonces, la nueva situación social será peor; y si tanto, el primer como la segundo principio son posibles, o bien, imposibles, entonces quien deba decidir -en este caso, el juez-, deberá abstenerse de efectuar una proposición de bienestar social, porque de otra forma, no podrá valorar cuál de tales estados sociales es mejor que el otro, pudiendo incurrir en la paradoja ${ }^{29}$. Lo anterior se muestra en la Tabla $\mathrm{N}$ o 1 :

TABLA 1

Posibilidad/imposibilidad de primer y segundo principio de Scitovszky

\begin{tabular}{|c|c|c|c|}
\hline \multirow{2}{*}{$\begin{array}{c}\text { Segundo } \\
\text { Principio }\end{array}$} & Posible & Posible & Imposible \\
\cline { 2 - 4 } & & $\begin{array}{c}\text { Paradoja/deber de } \\
\text { abstención }\end{array}$ & $\begin{array}{c}\text { Nuevo estado social es } \\
\text { peor que existente/Hicks }\end{array}$ \\
\cline { 2 - 4 } & Imposible & $\begin{array}{c}\text { Nuevo estado social } \\
\text { superior a existente/ } \\
\text { Kaldor con compen- } \\
\text { sación real }\end{array}$ & $\begin{array}{c}\text { Peor estado social posi- } \\
\text { ble/deber de abstención }\end{array}$ \\
\hline
\end{tabular}

Sin embargo, la posibilidad de juegos que ejecuten repetidamente ambos principios hasta llegar a la decisión social que evite la paradoja, no se puede replicar en el contexto de una decisión judicial ${ }^{30}$. El juzgador enfrentado a dos posibles estados sociales que emanen de su decisión (por ejemplo, estado social X si acoge la atribución mediata de ganadoresperdedores que sostiene $\mathrm{A}^{31}$, o bien, estado social Y si acoge la atribución mediata de gana-

\footnotetext{
28 SCITOVISKY (1941) pp. 86-87.

29 SCITOVisky (1941) p. 88.

30 Quizás si son posible de aplicar respecto de quienes elaboran políticas públicas u en otros contextos de decisión social, donde las posibilidades de repetir las propuestas regulatorias viene dada por la búsqueda de un nuevo estado social que mejore el existente.

31 Utilizando el factor de eficiencia Kaldor-Hicks, tenemos que, si bien la decisión judicial es la que genera los ganadores-perdedores, ella se basa en una elección previa entre diversas situaciones sociales que pueden estar en juego al momento de la decisión. Por lo mismo, podemos diferenciar la atribución inmediata de ganadores-
} 
dores-perdedores que sostiene B), no puede abstenerse de resolver por uno de ellos, en base a que tanto $\mathrm{X}$ como $\mathrm{Y}$ resultan eficientes al mismo tiempo.

En otras palabras, el juez no puede dejar de resolver absteniéndose entre las atribuciones mediatas de ganadores y perdedores $(\mathrm{X}, \mathrm{Y})$, cuando no conoce si está incurriendo en la paradoja por ser posible tanto el primer como el segundo principio de Scitovsky ${ }^{32}$.

Cabe señalar, por lo demás, que la valoración de los extremos de la paradoja, como de los principios que tienden a impedirla, no forman parte de los elementos de juicio que el juez debe considerar al momento de resolver un asunto, y sería tan poco realista sostener la necesidad de esa valoración, como pretender que el juez debe aplicar criterios de eficiencia conceptualizados en base al criterio Kaldor-Hicks, en base a una norma real o hipotética que diga "este asunto deberá resolverse de acuerdo al criterio Kaldor-Hicks". La reconstrucción de la eficiencia como factor de decisión social, por tanto, descansa sobre factores más realistas.

Con todo, el juez debe igualmente resolver ya que no se encuentra facultado para decidir "non liquet", declarando un asunto sin solución. La alternativa opuesta al "non liquet", corresponde al deber del juez de fallar ${ }^{33}$, cuyo reconocimiento en el sistema judicial chileno se concretiza en el deber de inexcusabilidad de rango constitucional en el artículo 76 de la Constitución Política de la República.

Así, no se encuentra dentro de las opciones posibles del juez una abstención de resolver el conflicto sometido a su decisión, en base a exigir que las partes modifiquen sus pretensiones de atribución mediata de ganadores-perdedores (aquellas que definen para el demandante y demandado los estados sociales X, Y, respectivamente), por el hecho de ser ambas atribuciones mediatas (suponiendo que no existen otras y que el juez tampoco puede considerar una tercera), eficientes al mismo tiempo según Kaldor-Hicks.

Adicionalmente, el juez debe resolver debido a que ejerce una función pública que impone la necesidad de decidir con efecto de cosa juzgada, a fin de otorgar seguridad jurídica y estabilidad a las normas jurídicas. En este sentido, se ha señalado que "la coerción es necesaria si el derecho pretende ser una práctica social que satisface sus propósitos formales

perdedores -que se alcanza por medio de la decisión judicial, y la atribución mediata de ganadores-perdedoresque viene dada por la elección de aquella opción disponible para el juez a efectos de adoptar la decisión judicial. En este sentido la atribución inmediata es única y pública, ya que consta en un acto jurídico procesal perfectamente identificable -resolución que contiene la decisión-, y además, dicho acto será de conocimiento de todos los sujetos, ya que normalmente los actos de los tribunales son públicos (En el caso del derecho chileno, ello encuentra su fuente en el artículo 8 de la Constitución Política de la República de 1980 y artículo 9 del Código Orgánico de Tribunales de 1943). En cambio, la atribución mediata tiene como característica ser múltiple y normalmente secreta, ya que, existen muchas posibles elecciones a ser consideradas por el tribunal para fundar una decisión eficiente en uso del criterio Kaldor-Hicks (todas aquellas que cumplan con los elementos de dicho concepto), y además, como normalmente la elección aceptada es aquella que se contiene en la sentencia el resto de las otras elecciones posibles de atribución mediata normalmente serán desconocidas para alguien distinto del juez.

32 No se incorpora el supuesto de imposibilidad de ambos principios, ya que en ningún caso se podrá considerar que dicho escenario constituye una mejora social a la existente. De esta forma, solamente cuando los dos principios que propone Scitovsky son posibles, puede configurarse el problema de la paradoja.

33 Martínez (2012) pp. 113-118. 
básicos definidos por los valores de la certeza jurídica y la eficacia" ${ }^{4}$, lo que se cumple, entre otras herramientas, por medio del ejercicio de la función jurisdiccional.

De esta forma, la aplicación de la posible paradoja (y de los principios elaborados para impedirla) en el contexto de la decisión jurisdiccional deviene en inabordable, por el cúmulo de información requerido para poder afirmar su existencia y por el hecho de encontrarnos ante un contexto de decisión judicial que impone la necesidad de resolución aun cuando sea posible la concurrencia de los dos principios que pretenden evitar la paradoja de Scitovsky. Como consecuencia de ello, la principal crítica al criterio Kaldor-Hicks, queda desestimada en el contexto del derecho procesal como contexto especial de decisión social, donde tiene aplicación el análisis económico del derecho.

Ahora bien, tal como hemos indicado, el proceso posee restricciones institucionales y en lo que aquí interesa nos referimos a la oportunidad única que tiene el juez para poder decidir. Esa oportunidad única es entendida en el proceso como un todo, más allá de que existirán instancias dentro del juicio (primera y segunda instancia), esto es, distintos estados de conocimiento de los hechos y del Derecho por parte de diversos tribunales. De esta forma, la idea de oportunidad única o no repetitiva, se traduce en la idea que generalmente en cada una de esas instancias del proceso, las posibilidades de decidir se reducen a un único y no repetitivo momento. Evidentemente, lo anterior, bajo la suposición que los jueces en cada instancia son distintos de los que conocieron el asunto en instancias anteriores ${ }^{35}$.

En este sentido, la decisión adoptada en el juicio como un todo, al producir efectos de cosa juzgada sustancial determina que el juez resolverá ese asunto particular (aquel conflicto en el que concurre la triple identidad con uno anterior ${ }^{36}$ ), una sola vez. La decisión una vez firme y ejecutoriada ${ }^{37}$, adquiere el carácter de inmutable tanto en ese juicio como para cualquier proceso posterior. El asunto no podrá volver a plantearse, existiendo una serie de instrumentos procesales tendientes a resguardar que el efecto de cosa juzgada se preserve $^{38}$. En tales consideraciones se basa la caracterización del proceso como un juego único y no repetido: la cosa juzgada sustancial le otorga dicho carácter.

\footnotetext{
34 Alexy (2008) p. 90.

35 En este sentido, las reglas de inhabilidad de los jueces impiden que estos puedan resolver de asuntos que ya conocieron previamente. Así ocurre en el sistema jurídico chileno, en razón de la causal de implicancia del artículo 195 N o 8 del Código Orgánico de Tribunales de 1943 y de la causal de recusación del artículo 196 N o 10 del Código Orgánico de Tribunales de 1943.

36 En efecto, en el derecho procesal civil chileno, la identidad entre dos procesos que permite el ejercicio de la excepción de cosa juzgada requiere la concurrencia de la triple identidad contenida en el artículo 177 del Código de Procedimiento Civil de 1902: identidad legal de persona; identidad de cosa pedida e identidad de causa de pedir. En el caso del derecho procesal civil español, el efecto de cosa negativo de la cosa juzgada, se encuentra regulado en el artículo $222 \mathrm{~N}$ o 1 de la LEY 1/2000, de 7 de enero, de Enjuiciamiento Civil.

37 En el caso del derecho procesal civil chileno el artículo 174 del Código de Procedimiento Civil de 1902 establece los supuestos en los cuales se entenderá firme y ejecutoriada una resolución. En el caso del derecho procesal civil español, esa materia se contiene en los artículos 207 y siguientes de la LeY 1/2000, de 7 de enero, de Enjuiciamiento Civil.

38 En este sentido, en el sistema procesal civil chileno son variadas las posibilidades de un demandado de fundarse en el efecto negativo de la cosa juzgada sustancial. Puede invocarla como excepción dilatoria, en base a los artículos 303 y 304 del Código de Procedimiento Civil de 1902; como excepción perentoria o de fondo en la contestación de la demanda, artículos 304 y 309 N o 3 del Código de Procedimiento Civil de 1902; puede
} 
Ahora bien, junto con la cosa juzgada sustancial concurre la cosa juzgada formal. Según Eduardo Couture, la cosa juzgada formal corresponde al efecto de las resoluciones que "Se cumplen y son obligatorias tan solo en relación al proceso en que se han dictado $(. . .)^{\prime 39}$. Esto es, la cosa juzgada formal corresponde a la cualidad de los efectos de una sentencia consistentes en su impugnabilidad dentro del proceso al haber precluido los medios recursivos que pudieron interponerse en su contra. La cosa juzgada formal normalmente dice relación con incidentes que se van promoviendo en el proceso, habitualmente resueltas por una sentencia interlocutoria, a la cual la legislación otorga también el efecto de cosa juzgada ${ }^{40}$.

La lógica de ello es que la cosa juzgada formal es un antecedente de la cosa juzgada sustancial. Por lo mismo, cabe afirmar que respecto de cada asunto que conforma un incidente, esto es, respecto de cada cuestión accesoria a lo principal que es resuelto por una sentencia interlocutoria, aparece la plena adecuación de la caracterización del proceso como una secuencia de juegos únicos sucesivos. Por ello, al proceso le interesa como concatenación de actos hacia un fin determinado, ir cerrando las cuestiones anexas, de la misma forma que le interesa que la cuestión principal no sea discutida nuevamente. En ambos casos, en sus contextos particulares, se encuentra presente la idea del juego único y no repetitivo.

Sin embargo, excepcionalmente es posible que un asunto vuelva a plantearse a pesar de haber sido resuelto con efecto de cosa juzgada. Cuando ello ocurre, hablamos de cosa juzgada sustancial provisional y cosa juzgada formal provisional. La novedad lo representa la "provisionalidad" con la que opera la cosa juzgada en alguno de esos supuestos. Este elemento de "provisionalidad" significa que varían las circunstancias tomadas en consideración al momento de dictar una sentencia que originalmente produjo efecto de cosa juzgada sustancial o formal. Excepcionalmente, el legislador autoriza que dada la entidad y relevancia social de esa variación de circunstancias, sea posible un nuevo juicio a pesar de la cosa juzgada sustancial producida originalmente (cosa juzgada sustancial provisional), o bien, sea posible que una decisión intermedia adoptada en el curso de un proceso pueda ser planteada nuevamente en el proceso en cuestión (o cosa juzgada formal provisional).

Explicando el elemento de "provisionalidad" en el contexto anterior, Abraham Vargas, señala que: "en cuanto al valor de cosa juzgada, como medida provisional que es, carece del mismo, lo que significa que no tenga ninguna fuerza para vincular. Por el contrario, vincula a las partes y al propio juez, quien no podrá modificarla, salvo que haya un cambio en las circunstancias que así lo justifique" ${ }^{31}$.

alegarse en cualquier estado del proceso como excepción mixta, artículo 310 del Código de Procedimiento Civil de 1902; puede invocarla como causal de acumulación de autos para evitar efectos de cosa juzgada incompatibles, artículo $92 \mathrm{~N}$ o 3 del Código de Procedimiento Civil de 1902; como causal de casación en la forma, de conformidad al artículo $768 \mathrm{~N}$ o 6 del Código de Procedimiento Civil de 1902, y finalmente, y como causal de recurso de revisión si es que no se ha opuesto en el curso del proceso, de acuerdo al artículo $810 \mathrm{~N}$ o 4 del Código de Procedimiento Civil de 1902.

39 Couture (2004) p. 339.

40 Artículo 175 del Código de Procedimiento Civil de Chile de 1902.

41 Vargas (1999) p. 64. 
Lo relevante del elemento de provisionalidad de la cosa juzgada es que en los casos excepcionales que ella se plantea, el asunto principal o accesorio puede volverse a formular, destruyendo la caracterización del proceso como juicio único y no repetido principal o accesorio, dependiendo de si nos encontramos en presencia de cosa juzgada sustancial o formal.

Dos consecuencias se siguen de ello. La primera es que se produce un efecto deseable en términos de mayor información para el juzgador, sobre todo en los casos en que es el mismo juez quien vuelve a conocer del asunto anteriormente resuelto. En este sentido, el juez conocerá de un asunto en el juego repetido no solamente por segunda vez, y además lo conocerá con un trasfondo informacional mucho mayor, ya que sabrá cuáles fueron los efectos que produjo su decisión, y asimismo, con esa mayor información podrá visualizar prospectivamente si su decisión inicial fue correctamente adoptada. La segunda consecuencia vinculada con la anterior, también es un efecto deseable, y refiere que ese mayor cúmulo de información permitirá al momento de adoptar la nueva decisión -en el ejercicio del juego repetido- reducir los costos del error involucrado en la resolución judicial que se dicte.

Por lo mismo, la existencia de juegos repetidos en la medida que resulten excepcionales -de otra forma, afectarán la finalidad de certeza jurídica ínsita en las decisiones judiciales firmes- y bien delimitados, producirán consecuencias positivas desde una perspectiva de eficiencia.

La pregunta que también surge es si la provisionalidad que derrumba la caracterización del proceso como juego único, ¿Reintroduce la reiteración de decisiones sociales que generan la intransitividad del criterio Kaldor-Hicks?, de responderse afirmativamente esa pregunta, tendríamos que en los casos donde concurre provisionalidad, sería aceptable la crítica al criterio mencionado en razón de la paradoja de Scitovsky, y por tanto, la misma podría ser valorada por el juez al momento de decidir el asunto involucrado en el juego repetitivo.

Para descartar una respuesta afirmativa a la pregunta anterior, quisiera desarrollar un ejemplo acerca del rol de la provisionalidad como causante de juegos judiciales repetidos en el derecho procesal concursal, área del derecho que hemos considerado que constituye un subconjunto normativo donde se reconoce a la eficiencia como factor de decisión ${ }^{42}$.

Un ejemplo de ello lo encontramos en el artículo 88 de la Ley $\mathrm{N}$ o 20.720, de fecha 9 de enero de 2014, Ley de Reorganización y Liquidación de Empresas y Personas, que regula lo que he denominado la segunda chance de la reorganización ${ }^{43}$. En efecto, en la legislación mencionada, se otorga la posibilidad de volver a plantear un procedimiento concursal de reorganización, a pesar que haya fracasado una propuesta anterior. En este sentido, la única norma de clausura a una nueva propuesta de procedimiento concursal de reorganización se contiene en el artículo 88 de dicha normativa, cuando a propósito de la impugnación de un acuerdo de reorganización aprobado, se señala que la resolución que acoge esa impugnación en base a la causal $\mathrm{N}$ o 4 y $\mathrm{N}$ o 5 del artículo 85 de la Ley $\mathrm{N}$ o 20.720, impedirá que posteriormente pueda volver a plantearse un nuevo acuerdo de reorganización.

\footnotetext{
42 Ver pie de página No 2.

43 Carrasco y Núnez (2014) p. 61.
} 
Sin embargo, no existe impedimento para que se vuelva a plantear un nuevo procedimiento concursal de reorganización a pesar de concurrir las otras causales de impugnación ( $\mathrm{N}$ o 1, 2, 3 y 6 del artículo 85 de la Ley No 20.720), ni tampoco existe impedimento de doble chance del procedimiento concursal de reorganización en los casos en que tal procedimiento haya dejado de producir efectos por nulidad (artículo 97 de la Ley N o 20.720), o por incumplimiento (artículo 98 de la Ley No 20.720).

Me quisiera detener en la posibilidad de segunda chance del procedimiento concursal de reorganización en caso de concurrir incumplimiento a la primera reorganización alcanzada. La lógica detrás de esta segunda chance es que a pesar de la inobservancia del deudor a las exigencias de la reorganización, podrá eventualmente volver a plantearse un acuerdo de ese tipo. La pregunta que surge, ¿Dónde se encuentra el problema? la misma surge de que la opción inicial por un procedimiento concursal de reorganización supuso una elección entre esa alternativa y un procedimiento concursal de liquidación. En este sentido, en el marco de la decisión sobre si iniciar un procedimiento concursal de reorganización se debe considerar, por una parte, la viabilidad del acuerdo ${ }^{44}$, como asimismo, la alternativa de realización de los activos del deudor en un procedimiento concursal de liquidación ${ }^{45}$.

Pues bien, si inicialmente se optó por el procedimiento concursal de reorganización, entonces, se valoró de mejor forma el factor de viabilidad del acuerdo versus el factor de monto probable de liquidación de los activos del deudor. Evidentemente que esa decisión genera ganadores y perdedores, muy probablemente dentro de los primeros tendremos al deudor, y muy probablemente, dentro de los segundos tendremos a algunos acreedores.

Sin embargo, si el procedimiento concursal de reorganización se incumple ${ }^{46}$, esa valoración inicial resultó prospectivamente errada (existencia de costo de error concursal), toda vez que, el acuerdo de reorganización no era susceptible de ser cumplido.

Con todo, y dado que la existencia de un incumplimiento basado en el artículo 88 de la Ley $\mathrm{N}$ o 20.720 no impide que un acuerdo de reorganización vuelva a ser planteado, y es probable que si eso ocurre tendremos la posibilidad de valorar entre el factor de viabilidad del deudor y el factor de monto probable de realización de activos.

Lo relevante, y esta es la finalidad del ejemplo, es que al momento de decidir este segundo juego de decisión concursal, se tendrá como información disponible una serie de elementos que en la primera decisión no poseía, tales como, la conducta del deudor tendiente a dar cumplimiento o no al acuerdo de reorganización, la viabilidad de su empresa para dicho efecto, y la manera en que verificó el comportamiento de los acreedores.

De esta forma, en supuestos de juegos judiciales no únicos se producen tres consecuencias: (i) Primero, se hace más plausible que se pueda aplicar correctamente criterios

44 El artículo 58 No 8 letra a) de la LEY No 20.720, establece que el veedor deberá emitir un informe que incluya entre otros aspectos "Si la propuesta es susceptible de ser cumplida, habida consideración de las condiciones del deudor”.

45 El artículo 58 No 8 letra b) de la LEY No 20.270, dispone que el veedor también deberá informar acerca de "El monto probable de recuperación que le correspondería a cada acreedor en sus respectivas categorías, en caso de un Procedimiento Concursal de Liquidación".

46 Concurrencia del supuesto del artículo 98 de la LEY N o 20.720 de 2014. 
de eficiencia; (ii) Segundo, se reducen los costos del error, y (iii) Tercero, y más importante, respondiendo a la pregunta con que iniciamos el ejemplo del artículo 88 de la Ley No 20.720, no es exigible que quien adopte la decisión respectiva valore los principios que pretenden evitar la paradoja. En otras palabras, los supuestos excepcionales donde existen juegos repetitivos, no son de aquellos que buscan validar la crítica al criterio Kaldor-Hicks, por medio de aceptar la plausibilidad de la paradoja de Scitovsky, toda vez que, la repetición del juego no surge por la intransitividad de la atribución inmediata de ganadoresperdedores contenida en una decisión respecto a alguna otra atribución inmediata posible. Más bien la repetición del juego emana de hechos exógenos y posteriores a la atribución inmediata que permitió fundar la primera decisión, como por ejemplo, los supuestos de incumplimiento en el ejemplo analizado.

Considerando las conclusiones anteriores, uno podría tener una sensación de desamparo, que lo motivara a ampliar a los supuestos de provisionalidad, dada las consecuencias de eficiencia deseables que se siguen de ella. Sin embargo, no considero que eso constituya una alternativa justa. La certeza jurídica, como hemos visto, forma parte de la esencia de lo que entendemos como ejercicio jurisdiccional ${ }^{47}$, y no creo que los efectos positivos de la provisionalidad, logren siquiera compensar la pérdida que pudiera traer consigo debilitar la seguridad jurídica alcanzada para la generalidad de los casos donde operan los efectos de cosa juzgada sustancial y formal.

\section{EL ANÁLISIS ECONÓMICO DEL DERECHO COMO HERRAMIENTA METODOLÓGICA APLICABLE AL PROCESO EN SU FAZ DE DECISIÓN JUDICIAL: EL DERECHO PROCESAL COMO JUEGO ÚNICO Y VÍA DE INTERIORIZACIÓN}

En esta sección, me gustaría examinar una razón que coadyuva a atemperar el desamparo procesal que examinamos en el apartado anterior. Quisiera explorar consideraciones que favorecen el entendimiento del ejercicio jurisdiccional como una actividad que tienda a la seguridad jurídica, incluso en presencia de un cúmulo imperfecto de información. La idea de trasfondo supone entender el proceso civil como un juego único y no reiterativo que tiende a la interiorización de las expectativas de éxito y fracaso de las partes.

En efecto, el juego judicial único establece incentivos para alcanzar tal éxito, así como para aminorar comportamientos oportunistas de las partes. Al analizar lo anterior, nos centraremos en un aspectos del sistema judicial, cual es, la interacción de las partes que intervienen en el proceso ${ }^{48}$.

Por tanto, a continuación señalaremos las características del proceso como juego único, centrándonos en las razones por las cuales se generan incentivos para que las partes interioricen sus expectativas de éxito y fracaso. A esta razón la denominaremos "juego único procesal como vía para interiorizar cargas informativas". Ella conduce a demostrar que al

\footnotetext{
47 Ver pie de página No 34. En este mismo sentido, MarinOni et al. (2010) p. 110, 121-124.

48 PASTOR (1993) pp. 32-33.
} 
momento de la decisión el juez posee la información suficiente para poder resolver en base a consideraciones de eficiencia en aquellos subconjuntos normativos que admiten la procedencia del análisis económico del derecho procesal.

En este sentido, el proceso civil contempla en su configuración actual mecanismos para interiorizar de los costos asociados al cumplimiento de las cargas de prueba de las partes, en términos tales, que son estas las que racionalmente deberán satisfacer tales cargas. Lo anterior, incluso en el contexto en que la eficiencia sea un factor de decisión judicial, donde se supone que los niveles informacionales para fundar las decisiones son mayores.

Para tal efecto, supondremos que las personas demandan con el objeto de perseguir un fin útil para ellas mismas, esto es, que las personas que inician acciones legales no instrumentalizan el proceso con fines diversos a obtener una decisión favorable a sus intereses.

Tal suposición puede ser irrealista en ciertos casos, ya que en algunas oportunidades las personas inician acciones con miras a forzar una negociación, amedrentar a la parte contraria, o bien, por cualquier otro motivo distinto de obtener un pronunciamiento judicial sobre una pretensión legítima de condena, declarativa o constitutiva. Con todo, creo que esos casos son excepcionales y no contaminarán las conclusiones a las que arribaré. La suposición, por tanto, descansa en una racionalidad débil, referida a que en general las personas no están dispuestas a solventar los gastos de un juicio cuando ello no les reporta algún beneficio concreto en base a una pretensión procesal.

Lo señalado respecto de la naturaleza de la acción procesal se analiza desde una perspectiva abstracta, esto es, se entiende la acción procesal como un derecho autónomo y anterior al proceso. De esta forma, la acción procesal se concretiza en el juicio, no teniendo el demandante un derecho a obtener una condena, una declaración o la constitución de un nuevo estado ${ }^{49}$, sino que, solamente posee el derecho a impetrar el ejercicio de la actividad jurisdiccional con miras a alcanzar alguno de esos fines.

Pues bien, aceptando la suposición anterior, el derecho procesal consagra reglas adjetivas funcionales que les informan a las partes acerca de la manera en que podrán obtener un pronunciamiento jurisdiccional favorable a las pretensiones del demandante o a las defensas del demandado. Tales reglas adjetivas funcionales corresponden a las reglas de carga de la prueba o onus probandi. Para el profesor Jordi Nieva, las reglas de carga de la prueba corresponden a normas de última ratio indispensables en el derecho de la prueba, por medio de las cuales se ofrece una solución al juez para emitir una sentencia en el caso que no tenga suficientes elementos para decidir ${ }^{50}$.

Tales reglas determinan sobre qué parte recae la tarea de probar. Asimismo, a tales reglas se asocia una norma que explícita o implícita refiere el efecto que se sigue de no cumplirse esa carga.

Por ejemplo, supongamos que existe una regla de carga de prueba hipotética denominada, $\mathrm{N}_{1}$; y una regla explícita o implícita que señala los efectos que se siguen del incumplimiento de las reglas de carga de la prueba, a la que denominaremos, $\mathrm{In}_{1}$ :

$\left(\mathrm{N}_{1}\right)$, está conformado, a su vez, por las siguientes subreglas:

49 Puppio (2008) pp. 150-151.

50 Nieva (2011) pp. 91-96. 
$\left(\mathrm{N}_{1,1}\right)$ La parte que afirme un hecho tendrá que probarlo.

$\left(\mathrm{N}_{1,2}\right)$ La parte que niegue el hecho afirmado en $\left(\mathrm{N}_{1,1}\right)$ deberá probar la negación de ese hecho, y

$\left(\mathrm{N}_{1,3}\right)$ La parte que modifique el hecho afirmado en $\left(\mathrm{N}_{1,1}\right)$ deberá probar la modificación del hecho.

$\mathrm{Al}$ respecto, se descarta una subregla $\left(\mathrm{N}_{1,4}\right)$, que establezca que: la parte que niegue $\left(\mathrm{N}_{1,2}\right)$ o $\left(\mathrm{N}_{1,3}\right)$, deberá probar la negación del hecho afirmado en $\left(\mathrm{N}_{1,1}\right)$ o la negación de la modificación del hecho afirmado en $\left(\mathrm{N}_{1,1}\right)$; toda vez que, ello supondría, en la mayoría de las veces, la necesidad de probar la negación de la negación o modificación de un hecho afirmado, lo que se traduce en una prueba imposible. En efecto, la manera de probar la siguiente afirmación "Niego que el niega que me debe", se reconduce a $\left(\mathrm{N}_{1,1}\right)$, esto es, a la necesidad de probar que "debe".

Por su parte, $\left(\operatorname{In}_{1}\right)$, está conformada por las siguientes subreglas:

$\left(\mathrm{In}_{1,1}\right)$, Si la parte que afirma un hecho, no lo prueba, y no concurren los supuestos $\left(\mathrm{N}_{1,2}\right)$ y $\left(\mathrm{N}_{1,3}\right)$, entonces, el hecho afirmado en $\left(\mathrm{N}_{1,1}\right)$, se tendrá por no probado, $\mathrm{y}$

$\left(\mathrm{In}_{1,2}\right)$, Si la parte que niega o modifica el hecho afirmado en $\left(\mathrm{N}_{1,1}\right)$, no prueba esa negación o modificación, entonces, se tendrá por no probado el hecho negado o modificado en $\left(\mathrm{N}_{1,2}\right)$ y $\left(\mathrm{N}_{1,3}\right)$, respectivamente.

Por otro lado, las partes que participan del proceso poseen el siguiente universo informacional, al que denominaremos, I:

$\left(\mathrm{I}_{1}\right)$ Conocen las subreglas de la regla de carga de la prueba $\left(\mathrm{N}_{1,1}\right)$;

$\left(\mathrm{I}_{2}\right)$ Conocen las subreglas de la regla que explícita o implícita señala los efectos que se siguen del incumplimiento de las reglas de carga de la prueba $\left(\operatorname{In}_{1}\right)$;

$\left(\mathrm{I}_{3}\right)$ Conocen que tienen un proceso único para acreditar las afirmaciones, modificaciones o negaciones de hechos realizadas y soportar las consecuencias del incumplimiento de la carga de prueba;

$\left(\mathrm{I}_{4}\right)$ Conocen que están litigando en un subconjunto normativo determinado, en donde puede operar la eficiencia como factor de decisión $\left(\mathrm{I}_{4}\right)$, o bien, donde opera otro criterio decisional, como por ejemplo, la justicia o equidad $\left(\mathrm{I}_{4}^{*}\right)$.

$\left(\mathrm{I}_{5}\right)$ Conocen que tienen un único juicio para satisfacer sus expectativas de pretensión o defensas.

Como consecuencia de ese universo informacional las partes tienen todos los incentivos necesarios para que en el desarrollo del juego único puedan acreditar sus pretensiones o defensas. El juego único, por tanto, genera que las partes interioricen los costos de incurrir en $\left(\operatorname{In}_{1,1}\right)$ o $\left(\operatorname{In}_{1,2}\right)^{51}$.

51 Cabe señalar que las bondades informacionales de las reglas de carga de la prueba han sido reconocidas por algunos autores, como ocurre con la profesora Carmen Domínguez, quien citada por Pablo Carvajal, ha soste- 
Así, en primer lugar, desde la perspectiva de un litigante racional, si el costo de probar un hecho, al que denominaremos, $\mathrm{CN}_{\mathrm{X}, \mathrm{X}}$, es mayor o igual que el costo de no tener por probada la afirmación de ese hecho, al que notaremos, In $\mathrm{n}_{\mathrm{x}, \mathrm{x}}$; entonces, la afirmación del hecho no será probada:

$$
\left(\mathrm{CN}_{1,1}\right) \quad\left(\mathrm{CIn}_{1,1}\right)[1]
$$

Seguidamente, si el costo de probar la negación o modificación de un hecho (" $\mathrm{CN}_{\mathrm{X}, \mathrm{X}}$ ") es mayor o igual que el costo de no tener por probada la negación o modificación del hecho primeramente afirmado $\left(\operatorname{In}_{\mathrm{x}, \mathrm{x}}\right)$, entonces, la negación o modificación del hecho originalmente afirmado no será probada:

$$
\begin{gathered}
\left(\mathrm{CN}_{1,2}\right) \quad\left(\mathrm{CIn}_{1,2}\right)[2], \mathrm{o} \\
\left(\mathrm{CN}_{1,3}\right) \quad\left(\mathrm{CIn}_{1,2}\right)[3]
\end{gathered}
$$

Por lo mismo, cada parte analizará internamente si se encuentra en algunas de las ecuaciones [1], [2] o [3] antes descritas y adoptará la decisión que mejor convenga a sus intereses, en cuanto a soportar el costo que emana de tales situaciones: no teniendo por probado un hecho afirmativo, negativo o modificativo alegado; o bien, aceptar incurrir en mayor desembolsos con miras a acreditar el hecho, su negación o su modificación, a pesar que las consecuencias de no tenerlo por acreditado no posean un mayor valor que el costo de probar el hecho, su negación o su modificación (este supuesto supondría desconocer la racionalidad inicial).

Dos comentarios en relación a lo señalado.

El primero es que las afirmaciones de racionalidad que se encuentran implícitas en las ecuaciones [1], [2] o [3] antes descritas, son solo suposiciones, ya que bien podría un sujeto incurrir en mayores costos de probar un hecho $\left(\mathrm{CN}_{1,1}\right)$, a pesar que las consecuencias negativas de no probarlo $\left(\mathrm{CIn}_{1,1}\right)$ sean menores que el costo de probarlo. Ello puede ocurrir cuando un demandante requiere probar tres elementos de hecho $\left[\left(\mathrm{E}_{1}\right),\left(\mathrm{E}_{2}\right)\right.$ y $\left.\left(\mathrm{E}_{3}\right)\right]$ para obtener un fallo favorable, precisamente, porque esos tres elementos son los que refiere la norma aplicable al caso. Sin embargo, el demandante en su demanda agregó un hecho adicional no establecido en la norma $\left(\mathrm{E}_{4}\right)$, y que a mayor abundamiento permitiría sostener su pretensión. En este caso, el costo de probar el cuarto hecho $\left(\mathrm{CE}_{4}\right)$, no trae consigo ninguna consecuencia relevante, ya que el costo de no tenerlo por probado $\left(\mathrm{CIE}_{4}\right)$, no impedirá

nido que: "Con ello, en su opinión, se provee a las partes de una regla clara y previamente conocida, por cuya virtud se evita mayores controversias. De hecho, añadió, se reduce la litigiosidad al dar bases más claras para alcanzar una transacción; se permite un mejor cálculo de los riesgos en la contratación; y se permite una mejor preconstitución de pruebas para que las partes se precavan debidamente de un conflicto futuro" confrontar con Carvajal (2012) p. 566. 
que el demandante obtenga el juicio si es que prueba los tres hechos relevantes $\left[\left(\mathrm{E}_{1}\right),\left(\mathrm{E}_{2}\right)\right.$ y $\left(E_{3}\right)$ ]. Con todo, el demandante puede querer acreditar ese hecho adicional, en razón que quiere asegurar su resultado bajo todo respecto. Ello puede ser perfectamente racional en base a las creencias que puede tener el sujeto ${ }^{52}$, y a una eventual, adversidad al riesgo ${ }^{53}$.

La segunda apreciación que quisiera señalar es que el costo de acreditar un hecho puede no ser siempre valorable ex ante por el sujeto, en términos tales que dicho costo puede generarse en el curso del proceso, o bien, puede sufrir variaciones respecto del que inicialmente fue previsto, para el caso que haya existido esa previsión. Esto no es más que una consecuencia de lo que diremos en la ecuación [4] en cuanto a que el factor que refleja los costos de litigación, [C] será determinado durante el curso del proceso.

Con todo, y a pesar de lo señalado en el segundo comentario precedente, la idea de interiorización que adoptan los sujetos respecto de los elementos de conocimiento que componen el universo informacional [I], se traduce en que tales litigantes al momento de iniciar una acción o de ser emplazados, deberán ponderar cuál es el costo probable que supone afirmar un hecho, o bien, negarlo o modificarlo. En este sentido, deben ponderar el costo probable de cada una de esas cargas $\left[\left(\mathrm{C} \mathrm{p} \mathrm{N}_{11}\right),\left(\mathrm{C} \mathrm{p} \mathrm{N}_{12}\right)\right.$ y $\left(\mathrm{C} \mathrm{p} \mathrm{N}_{13}\right)$, donde $(\mathrm{p})$, representa el elemento de probabilidad de tales costos.

Para estos efectos, (p), es el costo probable de afirmar un hecho, negarlo o modificarlo. En este sentido, siguiendo a Rafael Mery ${ }^{54}$, el esquema básico supone que un potencial litigante neutral al riesgo decidirá acudir a la justicia sí y solo sí:

$$
\begin{gathered}
\mathrm{P}(\mathrm{W}-\mathrm{M}-\mathrm{Cl}+\mathrm{M})+(1-\mathrm{p})(\mathrm{W}-\mathrm{M}-\mathrm{Cl}) \geq \mathrm{W}-\mathrm{M} \\
\mathrm{P}(\mathrm{W}-\mathrm{M}-\mathrm{Cl}+\mathrm{M})+(1-\mathrm{p})(\mathrm{W}-\mathrm{M}-\mathrm{Cl}) \geq \mathrm{W}-\mathrm{M} \\
\mathrm{P}(\mathrm{W}-\mathrm{Cl})+(1-\mathrm{p})(\mathrm{W}-\mathrm{M}-\mathrm{Cl}) \geq \mathrm{W}-\mathrm{M} \\
\mathrm{PW}-\mathrm{PCl}+[(\mathrm{W}-\mathrm{M}-\mathrm{Cl})(-\mathrm{pW}+\mathrm{PM}+\mathrm{PCl}) \geq \mathrm{W}-\mathrm{M} \\
(\mathrm{PM}) \geq \mathrm{Cl} \\
\mathrm{P} \geq \frac{\mathrm{Cl}}{\mathrm{M}}
\end{gathered}
$$

La parte derecha de dicha fórmula corresponde al valor probable que el demandante obtendrá de ir a juicio. Dicha parte de la ecuación está conformada por dos subpartes, la primera de ellas [], corresponde al valor probable que el demandante obtendrá de ganar el juicio, en este sentido, la probabilidad de obtener un resultado favorable (P), se multiplica al resultado que surge de las operaciones que consideran la riqueza inicial del demandante (W), menos el valor que él ha perdido como consecuencia de la actividad del demandado (-M), menos los costos del proceso (-C), más la recuperación de lo reclamado (M); la segunda subparte del lado derecho de la ecuación [], corresponde al valor probable que el

\footnotetext{
52 ELSTER (1997) p. 54.
}

53 Lo anterior se impone en razón de la función de utilidad cóncava del adverso al riesgo en cuanto implica una preferencia por un resultado seguro. En este sentido: KAHNEMAN y TVERSKY (1984) pp. 341-342.

De esta forma, si un litigio constituye un contexto de ganancia posible, el adverso al riesgo tenderá a maximizar esa característica, actuando con miras a asegurar un resultado procesal (ganar el litigio). Una consecuencia de ello será que probablemente rinda mayor cantidad de prueba que la necesaria.

54 Mery (2006) pp. 4-5. 
demandante obtendrá de perder el juicio, donde la probabilidad de un resultado negativo para el actor (1-p) se multiplica al resultado de las operaciones que consideran la riqueza inicial del demandante (W) menos el valor que él ha perdido como consecuencia de la actividad del demandado (-M), menos los costos del proceso (-C). Por el otro lado, la parte izquierda de la ecuación representa la decisión del sujeto de no ir a juicio, y comprende la operación de sustracción de la riqueza inicial del demandante (W), menos el valor que él ha perdido como consecuencia de la actividad del demandado (-M).

Por lo mismo, el costo probable de afirmar un hecho, negarlo o modificarlo pertenece al costo de litigación $(\mathrm{C})$ en la ecuación [4], en términos que:

$$
\left[\left(\mathrm{CpN}_{11}\right)(\mathrm{C})\right][5]
$$

Entonces, (p), también pertenece al factor (P) en la ecuación [4]:

$[(\mathrm{p})(\mathrm{P})][6]$

De esta forma, se refleja $\left(\mathrm{CpN}_{11}\right)$ perteneciente al costo probable de litigación (PC) en la fórmula [4]:

$$
\left[\left(\mathrm{CpN}_{11}\right)(\mathrm{PC})\right][7]
$$

En suma, un demandante deberá decidir demandar considerando el valor de $\left(\mathrm{CpN}_{11}\right)$ como parte de los costos probables de litigación (PC). Por su parte, el demandado, considerando muy probablemente los resultados de las ecuaciones [2] y [3] decidirán qué costos de carga de prueba asumir, tomando en cuenta que también para él, el valor de los costos de carga de prueba son parte de sus costos probables de litigación.

En ese escenario, el conocimiento que ambas partes tienen acerca del factor informacional $\left(\mathrm{I}_{4}\right)$, esto es, su conocimiento acerca que están litigando en un subconjunto normativo donde opera la eficiencia como factor de decisión, se introducirá como un factor en la determinación de los costos anteriores. En efecto, la existencia del conocimiento de encontrarse en un contexto de litigación $\left(\mathrm{I}_{4}\right)$ puede significar un mayor o menor nivel de gasto que aquel que supondría el conocer que se encuentran en un contexto de litigación alternativo, por ejemplo $\left(\mathrm{I}_{4}^{*}\right)$, donde la eficiencia no es un factor de decisión.

Nuevamente opera una interiorización, ya que, normalmente el demandante será libre de demandar en un contexto $\left(\mathrm{I}_{4}\right)$ o $\left(\mathrm{I}_{4}^{*}\right)$. En ambos casos deberá asumir los costos que surjan de cada uno de esos contextos.

Por ejemplo, si existe un crédito debido, el potencial demandante tiene a su disposición la posibilidad de accionar (si se cumplen los requisitos) en un juicio concursal donde opera la eficiencia como factor de decisión, lo que es conocido por el potencial demandante, por aplicación de $\left(\mathrm{I}_{4}\right)$; o bien, puede accionar en un proceso ejecutivo civil donde no opera la eficiencia como factor de decisión, lo que es conocido por el potencial demandante, por aplicación de $\left(\mathrm{I}_{4}^{*}\right)$. 
Si es el caso que los costos de litigar (dentro de los que se encuentran los costos de acreditar los hechos que fundan sus pretensiones) son mayores en el proceso concursal -lo que es conocido por el potencial demandante por aplicación de $\left(\mathrm{I}_{4}\right)-$, que los costos de litigar en un proceso civil ejecutivo -lo que es conocido por el potencia demandante por aplicación de $\left(\mathrm{I}_{4}^{*}\right)-$, y de todas formas, el potencial demandante decide demandar en el proceso concursal, entonces, sabrá que para obtener un mejor resultado tendrá que invertir más. De otro modo sus expectativas de triunfo se verán truncadas por aplicación de $\left(\operatorname{In}_{1}\right)$. Lo anterior, salvo que busque otros objetivos, que escapan de las suposiciones asumidas precedentemente.

De esta forma, dado el universo informacional (I) que existe disponible por las reglas procesales adjetivas y sustantivas de los sistemas jurídicos, las personas al decidir involucrarse o ser emplazadas en un proceso en donde se resuelve en base a eficiencia, asumen ex ante e interiorizan el mayor costo que ello supone (si es efectivamente mayor que su alternativa).

Por tanto, la tarea del juez en el contexto decisional judicial civil, con información imperfecta, con costos administrativos mayores y con costos del error aumentados por un juego en general único no repetitivo, no nos debe llevar al desamparo de un ejercicio jurisdiccional de resultados frustrantes. Basta entender que las partes interiorizan el costo de involucrarse en un proceso, incluso cuando lo anterior concurre en un contexto de decisión donde se resuelve en base a eficiencia, y en donde, probablemente los elementos a considerar para el buen éxito del demandante sean mayores que su alternativa.

Las partes interiorizarán esos costos ponderando que tendrán una sola oportunidad para acreditar sus posiciones, por lo que el costo de cumplimiento de la carga de prueba $\left(\mathrm{CN}_{\mathrm{xx}}\right)$ se podrá distribuir a lo largo del juicio, pero en todo caso, se agotará en el proceso como juego único, no siendo de utilidad que las partes reserven argumentos o pruebas para después del proceso, porque su alternativa de probanza se habrá frustrado, ya que habrá operado la regla de incumplimiento de carga probatoria $\left(\operatorname{In}_{x, x}\right)$. El juego único induce a sincerar las pruebas que admiten un resultado favorable para el sistema jurídico. Si la parte no posee la forma de allegar esas pruebas en este juego único, entonces, hay justicia en el resultado que determina el rechazo de su pretensión o defensa.

Detrás de esa razón de juego único procesal como vía para interiorizar cargas informativas se encuentra el rechazo a la idea intuitiva de favorecer que las partes prueben sus elementos de pretensión o defensas. Por el contrario, el proceso civil no necesita ni debe pretender favorecer ninguna necesidad de prueba ${ }^{55}$, sino que, debe tratar como iguales a las partes, entendida esa igualdad como una posición neutral del juez frente a aquello que potencialmente allegarán al juicio en cumplimiento de sus cargas probatorias ${ }^{56}$. Si no se satisfacen esas cargas, las reglas de incumplimiento determinarán un resultado que siempre

\footnotetext{
55 En este sentido, las facultades de prueba de oficio del tribunal se encuentran en el proceso civil limitadas con la finalidad de evitar que por esa vía pueda favorecerse a algunas de las partes subsidiando la deficiente satisfacción de sus cargas probatorias. En este sentido: PICó I JunOY (1997) p. 132.

56 Esta es la posición del profesor Iván Hubner para quien “(...) la misión del juez de mantener la igualdad no pretende exigirle que utilice su iniciativa probatoria para lograr equilibrios procesales cuando los sujetos se encuentran en una posición asimétrica, habiendo uno débil y uno fuerte" confrontar con Hubner (2011) p. 54.
} 
vendrá acompañado de un mandato deontológico o un juicio normativo, incluso cuando se desestime la acción en razón que el demandante incurrió en el supuesto $\left(\mathrm{Ic}_{1,1}\right)$. Aún en ese caso, la sentencia desestimatoria entrega un juicio normativo o calificación deontológica de deber ser que refuerza la idea de trato igualitario del juez civil, como consideración de neutralidad jurisdiccional a las partes en sus expectativas de cumplir sus cargas de probanza ${ }^{57}$.

\section{CONCLUSIONES}

Como herramienta para el juez se sostiene la aplicación de la eficiencia como criterio de decisión judicial (conceptualizada en el criterio Kaldor-Hicks). En este sentido, se ha tratado de dar las razones por las cuales consideramos que dicho factor subsiste como criterio de decisión a pesar de la paradoja de Scitovsky. Tal crítica al criterio Kaldor-Hicks no resulta aplicable al proceso, en razón de los principios que rigen la labor de adjudicación, así como, por las limitaciones de acceso a información que el proceso impone al juez. Todo lo anterior, no permite que el juez se encuentre en condiciones de valorar los extremos de la intransitividad que supone esa paradoja.

Adicionalmente, el hecho que los contextos de decisión donde opera la eficiencia requieran mayor información no significa una imposibilidad para que el juez cumpla su función, toda vez que, existe una lógica de interiorización que recae en las expectativas de ganancia o pérdida de las partes y que permite que el juez pueda descansar en ellas. La pretensión de Kornhauser en cuanto a que una aplicación del análisis económico del derecho deviene en imposible para un juez ${ }^{58}$, en razón de un eventual exceso de información para decidir, desconoce la lógica en que opera el proceso, en donde el demandante, si desea ganar, tiene los incentivos para que valore sus posibilidades de éxito y, de ser positivas, demande. No hay allí, nada de imposible.

\section{BIBLIOGRAFÍA CITADA}

Aghion, Philippe; Hart, Oliver; Moore, John (1992): “The Economics of Bankruptcy Reform”, Journal of Law, Economics and Organization, vol. V, No 8-3: pp. 523-546.

Agüero, Francisco; García De Cortázar, Verónica; García, William (2013): “Control Jurisdiccional de la potestad normativa del Tribunal de Defensa de la Libre Competencia en materia de telecomunicaciones", Informe en Derecho REGCOM, No 26 (Santiago, Facultad de Derecho de la Universidad de Chile).

AlEXY, Robert (2008): El concepto y la naturaleza del derecho (trad. Carlos Bernal Pulido, Madrid, Marcial Pons).

Cabrillo, Francisco (1988): Quiebra y liquidación de empresas (Madrid, Unión Editorial).

57 En este sentido, utilizando la terminología de Soeteman existe un juicio normativo que expresa una evaluación y requiere de justificación (la relativa al incumplimiento de la carga probatoria). No se trata de supuestos donde no existe juicio normativo porque se deja de lado el asunto, por ejemplo, en casos de declaraciones de incompetencia, donde el juez suspende su evaluación normativa, u otros casos, donde derechamente no se emite juicio algo. Véase, Soeteman (1989) pp. 151 y ss.

58 Kornhauser (1980) p. 606. 
Carrasco, Nicolás (2010): "La contracautela: Una mirada desde el análisis económico del derecho procesal”, Revista de Derecho y Humanidades, N 16: pp. 171-184.

Carrasco, Nicolás (2015): "El Análisis Económico del Derecho como herramienta procesal en la sociedad post-crisis" II Congreso Processulus (La Coruña, Universidad de La Coruña, 27 de noviembre de 2015).

Carrasco, Nicolás (2016): "El derecho procesal civil como ámbito de aplicación del análisis económico del derecho". II Congreso Estado de la Ciencia del Derecho (Ciudad de México D.F, Universidad Nacional Autónoma de México, 20 de febrero de 2016).

Carrasco, Nicolás y NúñEz, Raúl (2015): "Análisis Económico de la Administración de Justicia ¿La justicia como bien público o privado?”, Revista Chilena del Derecho, vol. No 42-2: pp. 595-613.

Carrasco, Nicolás y Núñez, Raúl (2014): “Conceptos generales del Análisis Económico del Derecho Concursal”, en Carrasco, Nicolás y Núñez, Raúl (Coords.), Presente y Futuro del Derecho Procesal Concursal Chileno. Una revisión desde el Análisis Económico del Derecho (Santiago, Editorial LegalPublishing) pp. 3-87.

Carvajal, Pablo (2012): "Facilidad probatoria en el proyecto de un nuevo código procesal civil”, Revista Chilena de Derecho, vol. No 39-3: 565-604.

Coleman, Jules (1979-1980): "Efficiency, Utility, and Wealth Maximization”, Hofstra Law Review, vol. 8: pp. 509-551.

Coleman, Jules (2004): "Los Fundamentos del Bienestar" (trad. Santiago Montt), Revista de Derechos y Humanidades, $\mathrm{N}^{\circ}$ 10: pp. 17-46.

Cooter, Robert; Ulen, Thomas (2008): Derecho y Economía (trad. Eduardo Suarez, México, DF, Fondo de Cultura Económica, segunda edición en español).

Cornelli, Francesca y Felli, Leonardo (1997): "Ex ante efficiency of bankruptcy procedures", European Economic Review, vol. 41: pp. 475-485.

Couture, Eduardo (2004): Fundamentos del Derecho Procesal (Montevideo, Editorial B de F, Montevideo).

Elster, John (1997): EGOnomics (trad. Irene Cudich, Barcelona, Gedisa Editorial, primera edición).

Hart, Oliver (2000): "Different approaches to Bankruptcy" Disponible en: http://www. nber.org/papers/w7921. Fecha de consulta: 11 de enero de 2016.

Hicks, John (1939): “The Foundations of Welfare Economics”, The Economic Journal, vol. No 49-196: pp. 215-228.

Hicks, John (1941): “The Rehabilitation of Consumers'Surplus", Review of Economic Studies, vol. No 8-2: pp. 108-116.

HuBNER, Iván (2011): "La iniciativa probatoria del juez y la igualdad de armas en el proyecto de código procesal civil", Revista Ius et Praxis, año 17 No 2: 53-76.

Jhunjhunwala, Bharat (1974): "Kaldor-Hicks-Scitovszky Criteria: A postmortem", Southern Economic Journal, vol. No 40-3: pp. 493-496.

KaLDOR, Nicholas (1939): "Welfare Propositions of Economics and Interpersonal Comparisons of Utility”, The Economic Journal, vol. 49 No 195: pp. 549-552.

Kahneman, Daniel y Tversky, Amos (1984): “Choices, Values, and Frames”, American Psychologist, vol. 39 No 4: pp. 341-350. 
Kornhauser, Lewis (1980): "A Guide to the Perplexed Claims of Efficiency in the Law", Hofstra Law Review, vol. 8 No 3: pp. 591-639.

Marinoni, Luiz; Pérez-Ragone, Álvaro y NúÑez, Raúl (2010): Fundamentos del Proceso Civil. Hacia una teoría de la adjudicación (Santiago, Abeledo Perrot).

Martínez, Patricio (2012): "El principio de inexcusabilidad y el derecho de acción desde la perspectiva del Estado Constitucional”, Revista Chilena de Derecho, vol. 39 No 1: pp. 113-147.

Mery, Rafael (2006): "Una aproximación Teórica y Empírica a la Litigación Civil en Chile” Disponible en: http://works.bepress.com/rafael_merynieto/8/. Fecha de consulta 2 de junio de 2016.

MontT, Santiago (2010): "El TDLC como ente regulador del comercio", Documento de Trabajo REGCOM, No 1 (Santiago, Facultad de Derecho de la Universidad de Chile).

Nieva, Jordi (2011): "Los sistemas de valoración de la prueba y la carga de la prueba: Nociones que precisan revisión”, Justicia: Revista de Derecho Procesal, vol. 3 No 4: pp. 91120.

Pastor, Santos (1993): ¡Ah de la Justicia! Política Judicial y Economía (Madrid, Editorial Civitas).

Peinado, Juan Ignacio (2006): “Cooperación y pillaje en el concurso”, en Peinado, Juan Ignacio y Valenzuela, Francisco Javier (Coords.), Estudios de Derecho Concursal (Madrid, Marcial Pons) pp. 231-257.

Picó I Junoy, Joan (1997): Las garantías constitucionales del proceso (Barcelona, Bosch Editor).

Puppio, Vicente (2008): Teoría General del Proceso (Caracas, Universidad Católica Andrés Bello).

Scitovisky, Tibor (1941): "A note on Welfare Propositions in Economics", Review of Economics Studies, vol. 9 No 1: pp. 77-88.

Sierra Alta-Ríos, Aníbal (2010): "Breves liminares sobre derecho y teoría de juegos", Revista de la Masestría en Derecho Económico, vol. 6 No 6: pp. 359-385.

Soeteman, Arend (1989): Logic in Law. Remarks on Logic and Rationality in Normative Reasoning, especially in Law (Dordrecht, Kluwer).

TAPIA, Javier y CORDERO, Luis (2015): "La revisión judicial de las decisiones regulatorias: Una mirada institucional", Revista Estudios Públicos, No 139: pp. 7-65.

TARuffo, Michele (2002): La prueba de los hechos (trad. Jordi de Ferrer, Madrid, Editorial Trotta).

TARUfFo, Michele (2003): "Algunas consideraciones sobre la relación entre prueba y verdad" Disponible en: http://www.cervantesvirtual.com/nd/ark:/59851/bmckk9s2. Fecha de consulta: 26 de abril de 2016.

Vargas, Abraham (1999): Estudios de Derecho Procesal (Mendoza, Editorial Jurídicas Cuyo).

VON BÜLOW, Oskar (2008): La teoría de las excepciones procesales y los presupuestos procesales (Buenos Aires, Librería El Foro). 
CARRasco Delgado, Nicolás — "El proceso civil como juego no repetitivo y como vía para interiorizar..."

\section{NORMAS CITADAS}

Código Orgánico de Tribunales chileno (15 de julio de 1943).

Código de Procedimiento Civil chileno (30 de agosto de 1902).

Constitución Política de la República de Chile (1980)

DFL No 70, de fecha 30 de diciembre de 1988, del Ministerio de Obras Públicas.

LEY No 20.720 (9/01/2014), Ley de Reorganización y Liquidación de Empresas y Personas.

LEY 1/2000, de 7 de enero, de Enjuiciamiento Civil. 